\title{
THE EXISTENCE OF OUTER AUTOMORPHISMS OF SOME NILPOTENT GROUPS OF CLASS 2
}

\author{
EUGENE SCHENKMAN ${ }^{1}$
}

In a recent conversation with $F$. Haimo the question arose as to whether a nilpotent group always possesses an outer automorphism. The object of this note is to show that the answer is in the affirmative for certain nilpotent groups of class 2 and also to show that if the group is finite but not Abelian, then for all primes $p$ when $p^{k}$ divides the group order it also divides the order of the group of automorphisms.

Some preliminary remarks. We let $G^{\prime}$ stand for $[G, G]$ the commutator subgroup of $G$; i.e. the group generated by all commutators $[a, b]=a b a^{-1} b^{-1}$ where $a$ and $b$ are elements of $G$; and also note that nilpotent of class 2 means that $G^{\prime}$ is in the center of $G$. From this last fact we readily obtain

$$
\begin{aligned}
{[a, b c] } & =[a, b][a, c], \\
{[a b, c] } & =[a, c][b, c], \\
{\left[a^{m}, b^{n}\right] } & =[a, b]^{m n}, \\
{[a, b] } & =[b, a]^{-1} .
\end{aligned}
$$

$E$ will denote the identity subgroup, $e$ the identity element of $G$.

We let $G(n)$ denote the subgroup of $G$ generated by the $n$th powers of the elements of $G$ and assume that for some prime $p$ there is an integer $k$ such that $G\left(p^{k}\right) \subset G^{\prime}$.

We shall begin with some general results probably well known (cf. for instance [1]), but we have included the proofs for completeness.

THeOREM A. If $G$ is an Abelian group such that, for some prime $p$ and integer $k, G\left(p^{k}\right)=E$, then $G$ is the direct product of cyclic groups.

Proof. If $k=1$ the theorem is true since $G$ is a vector space over the field of $p$ elements. We proceed by induction on $k$ assuming that $G(p)$ is a direct product of cyclic groups, $G(p)=\Pi \otimes\left(x_{\alpha}\right)$ where $\left(x_{\alpha}\right)$ designates the cyclic group generated by $x_{\alpha}$.

Let $y_{\alpha}$ be such that $y_{\alpha}=x_{\alpha}^{1 / p}$. Then the $y_{\alpha}$ generate a group $G_{1}$ which is a direct product, $G_{1}=\prod \otimes\left(y_{\alpha}\right)$. For $\prod y_{\alpha}^{n_{\alpha}}=e$ implies that $\Pi y_{\alpha}^{m_{\alpha}}$ $=\prod x_{\alpha}^{n_{\alpha}}=e$ whence $x_{\alpha}^{n_{\alpha}}=e$ for all $\alpha$, and hence $n_{\alpha}$ is a positive power

Presented to the Society, April 23, 1954; received by the editors March 2, 1954.

1 This research was supported by the U. S Air Force under contract number AF 18(600)-790 monitored by the Office of Scientific Research. 
of $p$; it follows that $\prod x_{\alpha}^{n_{\alpha} / p}=e$, whence $x_{\alpha}^{n_{\alpha} / p}=e$, and finally $y_{\alpha}^{n_{\alpha}}=e$.

Now let $G_{0}$ be the maximum subgroup of $G$ such that $G_{0}(p)=E$; then there is a subgroup $Q=\prod \otimes z_{\beta}$ such that $G_{0}=\left(G_{0} \cap G_{1}\right) \otimes Q$ and finally $G=G_{1} \otimes Q=\left(\Pi \otimes y_{\alpha}\right) \otimes\left(\Pi \otimes z_{\beta}\right)$ as can readily be verified.

By a similar method of proof we can obtain the following result.

Theorem B. If $G$ is Abelian, $G\left(p^{k}\right)=E$, and if $g_{1}, \cdots, g_{n}$ are not in $G(p)$ and if the group they generate is a direct product $\left(g_{1}\right) \otimes \ldots$ $\otimes\left(g_{n}\right)$, then there is an $H$ such that $G=H \otimes\left(g_{1}\right) \otimes \cdots \otimes\left(g_{n}\right)$.

Letting as usual $\Phi(G)$ denote the intersection of all maximal subgroups of $G$, we have the following result.

Theorem C. If $G$ is nilpotent such that $G\left(p^{k}\right) \subset G^{\prime}$, then $\Phi(G)$ $=\left\{G^{\prime}, G(p)\right\}$, the subgroup of $G$ generated by $G^{\prime}$ and $G(p)$.

Proof. $\Phi(G) \supset G^{\prime}$ by Theorem 12, p. 114, of [3] and by the same type of argument $\Phi(G) \supset G(p)$. On the other hand if $g$ is not in $\left\{G^{\prime}, G(p)\right\}$, then by Theorem B there is a maximal subgroup of $G$ not containing $g$, and hence $g$ is not in $\Phi(G)$.

\section{Some lemmas on automorphisms.}

Lemma 1. If $M$ and $H$ are subgroups of $G$ so that for $m \in M, h \in H$, $[m, h]=e$, and if $G=M H$, then any automorphism $\sigma$ of $H$ which leaves $M \cap H$ elementwise fixed can be extended to be an automorphism of $G$.

Proof. If $g$ is in $G$ then $g=m h$ where $m \in M, h \in H$, and $g^{\sigma}$ will be defined to be $m h^{\sigma}$. This defines $g^{\sigma}$ uniquely; for if $g=m_{1} h_{1}=m_{2} h_{2}$, then $m_{2}^{-1} m_{1}=h_{2} h_{1}^{-1}=\left(h_{2} h_{1}^{-1}\right)^{\sigma}=h_{2}^{\sigma}\left(h_{1}^{-1}\right)^{\sigma}$ whence $m_{1} h_{1}^{\sigma}=m_{2} h_{2}^{\sigma}$.

We next check that $\left(m_{1} h_{1}\right)^{\sigma}\left(m_{2} h_{2}\right)^{\sigma}=\left(m_{1} h, m_{2} h_{2}\right)^{\sigma}$. This can be seen since the left member reduces to $m_{1} h_{1}^{\sigma} m_{2} h_{2}^{\sigma}=m_{1} m_{2} h_{1}^{\sigma} h_{2}^{\sigma}$ and the right member to $\left(m_{1} m_{2} h_{1} h_{2}\right)^{\sigma}=m_{1} m_{2} h_{1}^{\sigma} h_{2}^{\sigma}$.

LEMMA 2. If $M$ is a normal subgroup of an arbitrary group so that the coset $a M$ is of order $n$ and so that $G=M(a)$, and if $z$ in $M$ is in the center of $G$ such that $z^{n}=e$, then the mapping $\sigma$ defined by the rule $\left(m a^{r}\right)^{\sigma}=m a^{r} z^{r}$ is an automorphism of $G$.

The verification is left to the reader.

In what follows we let $G^{\prime}$ be in the center of $G$ and let $G$ be generated by $a, b, c, \cdots, f$ such that $G / G^{\prime}$ is the direct product of $\left(a G^{\prime}\right)$, $\left(b G^{\prime}\right), \cdots,\left(f G^{\prime}\right)$ whose orders are $k_{a}, k_{b}, \cdots, k_{f}$, so that every element of $G$ is expressed uniquely as $w a^{r_{a}} b^{r b} \ldots f^{r_{f}}$ where $w \in G^{\prime}$ and $0 \leqq r_{a}<k_{a}, \cdots, 0 \leqq r_{f}<k_{f}$. We then have the following result.

Lemma 3. If $z$ commutes with $b, c, \cdots, f$ and the order of $a z$ is the 
same as the order of $a$, then the mapping $\sigma$ sending $g_{1}=w_{1} a^{\text {ra }} \cdots f^{\text {rs }}$ into $w_{1}(a z)^{r_{a}} \cdots f^{r s}$ is an automorphism of $G$.

Proof. Clearly $G^{\prime}$ is left elementwise fixed by $\sigma$. If now $g_{2}=w_{2} a^{s a}$ $\cdots f^{s b}$, then $g_{1} g_{2}=w_{1} w_{2}\left[b^{r b} \cdots f^{r s}, a^{s_{a}}\right] a^{r_{a}+s_{a}} b^{r b} \cdots f^{r s} b^{s b} \cdots f^{s s}$; and $\left(g_{1} g_{2}\right)^{\sigma}=w_{1} w_{2}\left[b^{r b} \cdots f^{r s}, a^{s a}\right](a z)^{r a+s a} b^{r b} \cdots f^{r s} b^{s b} \cdots f^{s s}$. But

$$
\begin{aligned}
\underset{g_{1} g_{2}}{\sigma} & =w_{1}(a z)^{r a} b^{r b} \cdots f^{r f} w_{2}(a z)^{s a} b^{s b} \cdots f^{s f} \\
& =w_{1} w_{2}\left[b^{r b} \cdots f^{r s},(a z)^{s a}\right](a z)^{r a+s a} b^{r b} \cdots f^{r s} b^{s b} \cdots f^{s f}
\end{aligned}
$$

and hence $\sigma$ is an automorphism since $\left[b^{r b} \cdots f^{r s}, a^{s a}\right]=\left[b^{r b} \cdots f^{r s}\right.$, $(a z)^{s_{a}}$ ] by the assumption on $z$ and by (1a) and (1c).

LemMa 4. Let $\Phi(G)$ be the $\Phi$ subgroup of the finite p-group $G$ and let $A$ be the group of automorphisms of $G$. Then the normal subgroup $N$ (cf. [3, p. 48]) of $A$ of all the automorphisms leaving every coset of $G$ with respect to $\Phi(G)$ fixed is a p-group.

Proof. There is a series of characteristic subgroups of $G, G$ $=G_{1}, G_{2}, \cdots, G_{n} \neq E, G_{n+1}=E$, such that $G_{i+1}$ is the group generated by $\left[G_{i_{0}}, G\right]$ and $G_{i}(p)$ where $i_{0}$ is the largest number less than or equal to $i$ so that $G_{i_{0}}$ is a member of the descending central series.

Now let $\sigma$ be an automorphism of $G$ so that $a^{\sigma}=a \phi_{a}$ where $\phi_{a} \in \Phi(G)$. Then since $\Phi(G)=G_{2}$ by Theorem $\mathrm{C}$, the $\Phi$ subgroup of $G / G_{n}$ is $\Phi / G_{n}$ and hence by an induction argument there is a power of $p$, namely $p^{k}$, so that $a^{\sigma p^{k}}=a z_{a}$ where $z_{a}$ is in $G_{n}$. But if $\tau$ is any automorphism of $A$ so that $a \tau=a z_{a}$ with $z_{a}$ in $G_{n}$ and hence in the center of $G$, then $\tau^{p}=1$; for $z_{a}$ is a product of commutators and pth powers and hence $z_{a}^{\tau}=z_{a}$ since each commutator and each $p$ th power is fixed under $\tau$ as is readily checked. Hence $a^{r^{p}}=a$ and $\sigma^{p^{k+1}}=1$. Thus every element of $N$ is of $p$-power order and the lemma is proved.

\section{The main theorems.}

THEOREM 1. If $G$ is a finite non-Abelian group of prime power order whose commutator subgroup is in the center, then the order of $G$ divides the order of the group of automorphisms of $G$.

Proof. Let $a, b, \cdots, f$ be generators of $G$ with the properties stated in connection with Lemma 3 , and so arranged that $[a, b]=w_{1}$ is an element of maximum order $m_{1}$ in $G^{\prime}$. Let $w_{1}, \cdots, w_{n}$ of orders $m_{1}, \cdots, m_{n}$ be a basis for $G^{\prime}$ so chosen that $m_{1} \geqq m_{2} \geqq m_{i}$ for $i=3$, $\cdots, n$. Then the order of $G$ is $m_{1} m_{2} \cdots m_{n} k_{a} \cdots k_{f}$.

Now if $d$ is one of the chosen generators and if $m_{1}$ divides $k_{d}$, then for $w$ in $G^{\prime}$ the map sending $g=w a^{r a} \cdots d^{r d} \cdots f^{r s}$ into $w a^{r a} \cdots$ $\left(d \cdot d^{t m_{1}}\right)^{r_{d}} \cdots f^{r f}$ for $t=0,1, \cdots, k_{d} / m_{1}$ is an automorphism by 
Lemma 3 which leaves the subgroup $(d)$ invariant. There are $k_{d} / m_{1}$ such automorphisms for the generator $d$.

By Lemma 2 there is an automorphism sending $w a^{r_{a}} \cdots d^{r_{d}}$ $\cdots f^{r s}$ into $w a^{r_{a}} \cdots\left(d w_{j}^{u q_{j}}\right)^{r_{d}} \cdots f^{r s}$ where $q_{j}=\max \left(1, m_{j} / k_{d}\right)$ and $u=0,1, \cdots, m_{j} / q_{j}$. There are min $\left(k_{d}, m_{j}\right)$ such automorphisms for the generator $d$ and for $j=1, \cdots, n$.

We note now that $c, \cdots, f$ can be so chosen that they commute with $a$ and $b$ modulo $\left(w_{2}\right) \otimes \cdots \otimes\left(w_{n}\right)$. For if $d$ is one of the generators $c, \cdots, f$ suppose $[a, d] \equiv[a, b]^{s}$ and $[d, b] \equiv[a, b]^{t}$ modulo $\left(w_{2}\right) \otimes \cdots \otimes\left(w_{n}\right)$. Then $\left[a, d b^{m_{1-s}} a^{m_{1}-t}\right] \equiv e \equiv\left[d b^{m_{1-s}} a^{m_{1-l} t}, b\right]$ and $d b^{m_{1-s}} a^{m_{1-t}}$ can replace $d$ as the generator with the required property.

Now if $q=\max \left(p, k_{b} / k_{a}, m_{2}\right)$, then $b^{q}$ commutes with $b, c, \cdots, f$; then for $u=0,1, \cdots, k_{b} / q$ there are $k_{b} / q$ elements $a b^{u q}$ and since the orders of these are powers of $p$ between $k_{a}$ and $k_{a} m_{1}$, there are $h+1$ possibilities for the orders where $p^{h}=m_{1}$. Hence by replacing $a$ by one of the $a b^{u q}$ if necessary there are by Lemma 3 at least $k_{b} / q(h+1)$ distinct automorphisms sending $g=w a^{r_{a}} \cdots f^{r s}$ into $w\left(a b^{u q}\right)^{r a} \cdots f^{r b}$. Similarly if $r=\max \left(p, k_{b} / k_{a}, m_{2}\right)$, interchanging the roles of $a$ and $b$ there are at least $k_{a} / r(h+1)$ more distinct automorphisms.

All of the above automorphisms are in the normal subgroup of the group of automorphisms of $G$ described in Lemma 4 which will then be at least of order $k_{a} \cdots k_{f}\left(m_{2} \cdots m_{n}\right)^{2} m_{2} x y$ where $x$ and $y$ are the least powers of $p$ greater than $k_{b} / q(h+1)$ and $k_{a} / r(h+1)$ and where $\left(m_{2} \cdots m_{n}\right)^{2} m_{2}$ is 1 if $G^{2}$ is cyclic.

But this order is as large as the order of $G$ if $m_{2}^{2} x y \geqq m_{1}$, which is true except for $m_{1}=8,16,32$ and 64 when $m_{2} \geqq p$. For then $m_{2}^{2} \geqq m_{1}$ unless $m_{1} \geqq p^{3}$; but in this case $p^{\left(m_{1}\right)^{1 / 2}}>m_{1} p=p^{h+1}$ whence $\left(m_{1}\right)^{1 / 2}$ $>h+1, m_{1} / m_{2}>\left(m_{1} / m_{2}^{2}\right)^{1 / 2}(h+1)$, and finally $x$ and $y$ being both greater than or equal to $m_{1} /\left[m_{2}(h+1)\right]$ we see that $x y \geqq m_{1} / m_{2}^{2}$.

We consider now the case where $m_{2}=1$ and first let $m_{1}=p^{2 k}$ for $k=1,2,3, \cdots$. Then $p^{k}>h+1$ (except when $m_{1}=4,9$, and 16) and $p^{2 k-1} /(h+1)>p^{k-1}$ whence $x$ and $y$ are greater than or equal to $p^{k}$ and $x y \geqq m_{1}$. Next let $m_{1}=p^{2 k+1}$ for $k=0,1,2, \cdots$; then except when $m_{1}=2$ or $8, p^{k+1}>(h+1)$ and $p^{2 k+1} / p(h+1)>p^{k-1}$ whence $x y \geqq m_{1} / p$. But by replacing 1 for $p$ in the expression for one of the numbers $r$, or $q$ by 1 , we can obtain one more automorphism of $p$ power order not in the subgroup of automorphisms already considered, which with that subgroup generates a $p$-group of order at least equal to that of $G$.

Hence we have proved the theorem except in the exceptional cases when $m_{1}=2,4,8,9$, or 16 when $m_{2}=1$; and $m_{1}=8,16,32$, or 64 when $m_{2} \geqq p$. 
For the proofs in these cases it is possible to apply Lemma 3. Thus for $m_{1}=2$, if $a^{2}$ and $b^{2}$ are in $G^{\prime}$ then two of the three elements $a, b$, and $a b$ have the same order; for definiteness let them be $a$ and $a b$. Then there is an automorphism of order 2 leaving $b$ fixed and sending $a$ into $a b$. If on the other hand $b^{2}$ is not in $G^{\prime}$, let $n$ be minimal so that $b^{n}$ is in $G^{\prime}$; then two of the elements $b, b a$, and $b^{n-2} b a=b b^{n-2} a$ have the same order and again there is an automorphism of order 2 not in the subgroup of automorphisms previously considered. Thus the theorem follows for $m_{1}=2$.

When $m_{1}=8$ if $a^{8}$ and $b^{8}$ are in $G^{\prime}$, then two of the elements $a, b$, $a b, a b^{2}$, and $a b^{3}$ have the same order and there is at least an automorphism of order 4 of the type holding $b$ fixed and sending $a$ into $a b$ or $a b^{2}$. If $b^{8}$ is not in $G^{\prime}$, then letting $n$ be minimal so that $b^{n}$ is in $G^{\prime}$ we see that two of the elements $b, b a, b^{3} a, b^{5} a$, and $b^{n-1} a$ have the same order and there is an automorphism of order at least 4 holding $b$ fixed and sending $a b$ into $a b^{3}$ or $a b^{5}$ or $a b^{n-1}$ (i.e., $a$ into $a b^{2}$ or $a b^{4}$ or $\left.a b^{n-2}\right)$. By a similar method, considering $b, b c, b c^{2}, b c^{3}$, and $b c^{4}$ where $c$ is a power of $a$ so that $c G^{\prime}$ has the same or lower order than $b G^{\prime}$, it is possible to find an automorphism of order at least 2 so that $a$ is fixed. Then the group consisting of these automorphisms together with those previously described has order at least equal to that of $G$, proving the theorem when $m_{1}=8$.

We omit the details of the few remaining cases since no new ideas are involved.

Corollary. If $G$ is a finite non-Abelian group whose commutator subgroup is in the center, then the order of $G$ divides the order of the group of automorphisms of $G$.

THEOREM 2. If $G$ is a p-group, if $G^{\prime}$ is in the center of $G$, and $G\left(p^{k}\right)$ $\subset G^{\prime}$, then $G$ has an outer automorphism.

Proof. We shall assume to the contrary that all the automorphisms of $G$ are inner and on the basis of this assumption will exhibit an outer autmorphism.

We shall suppose that $k$ is the smallest integer such that $G\left(p^{k}\right) \subset G^{\prime}$. Let $z$ in $G^{\prime}$ have maximum order $p^{r}$; then $r \leqq k$ since $G\left(p^{k}\right) \subset G^{\prime}$ implies $G^{\prime}\left(p^{k}\right)=E$ in view of (1c).

Now let $s$ be the smallest integer greater or equal to $r$ so that there is a $g \notin \Phi(G)$ such that $g^{p^{\natural}} \in G^{\prime}$. Then by Theorem B, $G=M(g)$ where $M$ is normal in $G$ and $G / M$ has order $p^{8}$. Hence Lemma 2 asserts that there is an automorphism, which is determined by an element $h$ since by assumption it is inner, such that $[h, g]=z$ and $[h, m]=e$ for $m \in M$. Now $M$ can be changed if necessary so as to con- 
tain $h$. For if $M(h)$ contains $M$ properly, then $M(h)$ contains $g^{\alpha}$ for some smallest number $q$, and then by Theorem B, $M(h)=\left(g^{q}\right) \otimes(h)$ $\otimes M_{1}$ and $G=(g) \otimes(h) \otimes M_{1}$ so that $(h) \otimes M_{1}$ has the desired property.

Now $h$ is not in $\Phi(G)$ since in that event, by Theorem C, $h$ would be of the form $\prod_{i} h_{1}^{p} g_{2}$ where $g_{2} \in G^{\prime}$, and then by (1b) and (1c), $[h, g]$ $=\left[\prod_{i} h_{i}^{p} g_{2}, g\right]=\prod_{i}\left[h_{i}, g\right]^{p}$, which would contradict the maximality of the order of $z$ in $G^{\prime}$ since the orders of $\left[h_{i}, g\right]$ are at most as great as that of $[h, g]=z$. Hence $G=N(h)$ where $N$ is normal in $G$ and $G / N$ has order $p^{t}$. But $p^{t}$ is at least equal to $p^{s}$ by the choice of $s$ and because of (1c) and the fact that $z$ has order $p^{r}$.

Again by Lemma 2 there is a $k$ so that, for $n \in N,[k, n]=e$ and $[k, h]=z^{-1}$ or $[h, k]=z$. Since $G=M(g), k=m g^{r}$ and $z=[h, k]$ $=\left[h, m g^{r}\right]=[h, g]^{r}=z^{r}$ whence $r=1$ and $k=m g$. Then $G=M(g)$ $=M(k)$.

Now if $P$ is the group generated by $h$ and $k$, then we shall show that $P / P^{\prime}$ is of order $p^{t+s}$. First $P^{\prime}=P \cap G^{\prime}$. For clearly $P^{\prime} \subset P \cap G^{\prime}$; on the other hand if $d \in P \cap G^{\prime}$ then by our assumption there is an $f$ such that $[f, k]=d$. But since $f=n h^{r}$ where $n$ is in $N,[f, k]=\left[h^{r}, k\right]$ $=[h, k]^{r} \in P^{\prime}$. Hence $P^{\prime}=P \cap G^{\prime}$.

Next we observe that if $P / P^{\prime}$ is of order less than $p^{r+8}$, then there must be a relation of the form $h^{p^{u}}=k^{p^{v}} \bmod G^{\prime}$ where $t>U \geqq V<S$. Then if $w=\left(k h^{-p^{u-v}}\right), w^{p^{v}}=k^{p^{v}} h^{-p^{u}} \in G^{\prime}$. But since $[h, w]=[h, k]$ $=z, w$ is not in $\Phi(G)$ for the same reason that $h \notin \Phi(G)$, and hence the existence of $w$ contradicts the way $s$ was chosen since $v<s$. We conclude that $P / P^{\prime}$ is of order $p^{t+s}$.

Let $Q=M \cap N$. Then, $\bmod G^{\prime}, Q$ has index $p^{t+8}$ in $G$; but $P$ has order $p^{t+s} \bmod G^{\prime}$. Furthermore $Q \cap P \subset G^{\prime}$ and hence $G=Q P$. Also $P^{\prime}=P \cap G^{\prime}$ so that $P^{\prime}=P \cap Q$. Finally $[q, p]=e$ for $q \in Q, p \in P$. Then by Theorem 1, $P$ has an outer automorphism leaving $P^{\prime}$ elementwise fixed; this can be extended to be an automorphism of $G$ by Lemma 1, and the proof of the theorem is completed.

It would be of interest to know whether Theorem 2 is valid if the class of nilpotency of the group is arbitrary.

\section{BIBLIOGRAPHY}

1. I. Kaplansky, Infinite Abelian groups, Ann Arbor, University of Michigan, 1954.

2. W. R. Scott, On the order of the automorphism group of a finite group, Proc. Amer. Math. Soc. vol. 5 (1954) pp. 23-24. 1949.

3. H. Zassenhaus, The theory of groups, trans. from the German, New York,

Louisiana State University aNd

The INSTITUTe for Advanced StUdy 